\title{
Overexpression of myosin VI regulates gastric cancer cell progression
}

Zishu Wang ${ }^{\text {a, }}{ }^{*}$, , Mingzhen Ying ${ }^{\text {b, } 1}$, Qiong Wu ${ }^{\text {a, }}$, Rui Wang ${ }^{\text {a }}$, Yumei Li ${ }^{\text {a }}$

${ }^{a}$ Department of Medical Oncology, First Affiliated Hospital of Bengbu Medical College, Bengbu, Anhui 233004, China.

b Department of Oncology, Changhai Hospital, the Second Military Medical University, Shanghai 200433, China

${ }^{1}$ Equal contributions.

* Corresponding authors: Zishu Wang and Qiong Wu Zishu Wang

Tel./Fax: +86-552-3074480, E-mail: zishuwang@163.com

Qiong $\mathrm{Wu}$

Tel./Fax: +86-552-3074480, E-mail: qiongwu68@aliyun.com

Running title: Oncogenic role of MYO6 in gastric cancer 


\section{Introduction}

Myosins are a group of actin-dependent $\mathrm{Mg}^{2+}$ ATPases that are best known for their roles in a wide range of eukaryotic motility processes, during which myosins use the energy produced by ATP hydrolysis to 'walk' along the actin filaments (Pollard and Korn, 1973; Krendel and Mooseker, 2005). The movement of myosins contributes to the transport of components and generation of contractile force (O'Connell et al., 2007; Olson and Sahai, 2009). Structurally, myosins contain three parts, a conserved N-terminal domain that binds actin and hydrolyzes ATP, a short neck domain that contains an IQ motif, and a C-terminal tail for carrying cargos (O'Connell et al., 2007). In the human genome, 40 myosin genes have been identified. Based on analysis of their head and tail domain structures, the 40 myosin genes can be divided into 12 classes (Berg et al., 2001). Only the class II myosins are called conventional myosins because they are known to form bipolar filaments and generate contractile force. The remaining myosins belong to unconventional classes which are generally thought to play a role in non-muscle cells (Wu et al., 2000; Berg et al., 2001; Dunn et al., 2006).

Myosin VI (MYO6), which was firstly identified in Drosophila, has unique cellular properties and functions, because it moves towards the minus end of actin filaments in the opposite direction to all other myosins (Wells et al., 1999). MYO6 is involved in multiple processes including vesicular membrane traffic, cell mobility, and mitosis (Buss et al., 2004). MYO6 comprises four different regions: the head domain, the $\alpha$-helical coiled-coil domain which mediates dimerization, the globular tail domain which binds specific target proteins, and the neck domain that regulates ATPase activity (Kellerman and Miller, 1992). Recently, MYO6 has been reported to play an important role in governing cancer progression in several cancers such as ovary cancer and prostate cancer (Yoshida et al., 2004; Dunn et al., 2006; Wei et al., 2008). In ovary cancer, loss of MYO6 in high-grade ovarian cancer cells suppressed cell migration in vitro (Yoshida et al., 2004). In prostate cancer, MYO6 was found to be over expressed in cancer tissues, and knockdown of MYO6 in LNCaP cells led to impaired in vitro cell migration and colony formation (Dunn et al., 2006). Further 
investigation showed knockdown of MYO6 significantly reduced the secretion of prostate-specific antigen (PSA) and vascular endothelial growth factor (VEGF), a growth factor important for tumor angiogenesis (Wells et al., 1999; Puri et al., 2010). However, the potential role of MYO6 in gastric cancers still remains poorly understood.

Therefore, in the present study, we try to clarify the function of MYO6 in gastric carcinogenesis via a short hairpin RNA (shRNA) lentivirus system. Functional analysis showed that MYO6 knockdown significantly inhibited cell proliferation and colony formation ability, led to G0/G1 phase cell-cycle arrest, and induced apoptosis, which suggests that MYO6 may play an important role in the tumorigenesis of gastric cancer in vitro.

\section{Materials and Methods}

\subsection{Cell culture}

Human gastric cancer cell lines, SGC-7901, AGS and MGC80-3, and human embryonic kidney cell line 293T (HEK293T) were purchased from the Type Culture Collection of the Chinese Academy of Sciences (Shanghai, China). AGS cells were grown in F-12 medium (Sigma) containing 10\% fetal bovine serum (FBS). SGC-7901 and MGC80-3 cells were grown in RPMI-1640 (HyClone) supplemented with 10\% FBS. 293T cells were grown in DMEM (HyClone) containing 10\% FBS. All cell lines were cultured at $37^{\circ} \mathrm{C}$ in a humidified incubator containing $5 \% \mathrm{CO}_{2}$.

\subsection{Oncomine cancer microarray database analysis}

The expression of MYO6 gene in gastric cancer was examined via Oncomine database (www.oncomine.com). Cui et al (GEO accession GSE27342) (Cui et al., 2011) and Wang et al dataset (GEO accession GSE19826) (Wang et al., 2012) datasets were used to compare expression levels of MYO6 between gastric cancer and normal tissues. DErrico et al (GEO accession GSE13911) (D'Errico et al., 2009) was used to compare the expression difference of MYO6 between normal tissues and two different types of gastric cancer including gastric intestinal type adenocarcinoma and gastric 
mixed adenocarcinoma. In addition, Chen et al (http://genome-www.stanford.edu/Gastric_Cancer2) dataset (Chen et al., 2003) was used to compare the differential expression of MYO6 between gastric intestinal type adenocarcinoma and normal tissues. The expression values of MYO6 were log-transformed, median-centered and normalized to one per array (Rhodes et al., 2004)

\subsection{Kaplan-Meier overall survival analysis}

The effect of MYO6 expression on the prognosis of 876 gastric cancer patients was analyzed using the Kaplan-Meier plotter online software (http://kmplot.com/analysis/). The Kaplan-Meier plotter can evaluate the effect of 54,675 genes on survival using 10,188 cancer samples including breast, lung, ovarian and gastric cancer patients. MYO6 expression and survival data from Affymetrix microarray (ID: 203216_s_at). To analyze the prognostic value of MYO6 gene, the samples were divided into two groups according to the median expression of MYO6. The two patient groups (high and low expression of MYO6) were compared via the Kaplan-Meier survival plot. The hazard ratio (HR) with 95\% confidence intervals (CI), and the log rank p value was computed as previously described (Gyorffy et al., 2010).

\subsection{Lentiviral vector construction and production}

$\begin{array}{lllll}\text { Two shRNA } & \text { sequences } & \text { (MYO6 } & \text { shRNA }\end{array}$ 5'-GTGAATCCAGAGATAAGTTTACTCGAGTAAACTTATCTCTGGATTCACTT TTT-3'; MYO6 shRNA (S2): 5'-CCAGATTTAACCATTCCATAAC TCGAGTTATGGAATGGTTAAATCTGGTTTTTT-3') targeting human MYO6 gene (NM_004999) and the negative control sequence (5'-GATCCTTCTCCGAACGTGTCACGTCTCGAGACGACGCACTGGCGGAGA ATTTTTG-3') were designed and constructed into the pFH-L vector (Shanghai Hollybio, China). The vectors containing target sequence or control non-targeting sequence were transfected into HEK293T cells, together with pCMV $\Delta 8.92$ and pVSVG-I packing vectors (Shanghai Hollybio, China) via Lipofectamine 2000 
(Invitrogen, USA) to generate lentiviruses, according to the manufacturer's instructions. After $72 \mathrm{~h}$, lentiviruses were harvested by purification and precipitation.

\subsection{Lentivirus infection}

Both AGS and MGC80-3 cells were cultured in 6-well plates with a concentration of $5 \times 10^{4}$ cells/well. For lentivirus infection, purified lentiviruses containing shRNA sequence targeting MYO6 (Lv-shMYO6(S1) or Lv-shMYO6(S2)) or control sequence (Lv-shCon) with the multiplicity of infection (MOI) of 30 for AGS cells or 60 for MGC80-3 cells, respectively. After 72 h, cells were observed by fluorescence microscopy to determine the infection efficiency by measuring the percentage of green fluorescent protein (GFP) positive cells.

\subsection{Quantitative real-time PCR ( $q R T-P C R)$ analysis}

Total RNA was extracted from gastric cancer cells by Trizol (Invitrogen, Carlsbad). The cDNA was synthesized from total RNA with random primers following the manufacturer's protocol (MBI Fermantas, Vilnius, Lithuania). Five sets of primers were as follows: MYO6, 5'-AATCACTGGCTCACATGCAG -3' (forward) and 5'-AATGCGAGGTTTGTGTCTCC-3' (reverse); cyclin A, 5'-GT TCCTCCTTGGAAAGCAAAC-3' (forward) and 5'-GGGCATCTTCACGCTCTAT TT-3' (reverse); cyclin D1, 5'-GCCCTCGGTGTCCTACTTC-3' (forward) and 5'-C CTCCTCGCACTTCTGTTCC-3' (reverse); p21, 5'-TCCAGCGACCTTCCTCATCC -3' (forward) and 5'-CATAGCCTCTACTGCCACCATC-3' (reverse); $\beta$-actin, 5'-GTGGACATCCGCAAAGAC-3' (forward) and 5'-AAAGGGTGTAACGCAACT A-3' (reverse). The PCR reaction system was $20 \mu \mathrm{L}$ mixtures $(10 \mu \mathrm{L} 2 \mathrm{X}$ SYBR premix ex-taq, $5 \mu \mathrm{L}$ cDNA, $0.8 \mu \mathrm{L}$ primers $(2.5 \mu \mathrm{M})$ and $\left.4.2 \mu \mathrm{LddH}_{2} \mathrm{O}\right)$. PCR cycle conditions were: $95^{\circ} \mathrm{C}$ for $1 \mathrm{~min}$ (initial denaturation), and 40 cycles of $95^{\circ} \mathrm{C}$ for $5 \mathrm{~s}$ and $60^{\circ} \mathrm{C}$ for $20 \mathrm{~s}$ (denaturation and extension). Fluorescence was analyzed by using the BioRad Connet Real-Time PCR platform (\#CFX96, Bio-Rad Laboratories, Inc., Hercules, CA, USA). All samples were examined in triplicate. The mRNA expression levels of MYO6 were normalized to that of $\beta$-actin mRNA by using comparative 
threshold cycle (ct) method, in which fold difference $=2^{-(\Delta \mathrm{ct} \text { of target gene- } \Delta \text { ct of reference })}$.

\subsection{Western blot analysis}

The whole cell protein was extracted from lentivirus-transduced cells using ice-cold protein lysis buffer (50 mM Tris-HCl, $150 \mathrm{mM} \mathrm{NaCl}, 1 \mathrm{mM}$ EDTA, 1\% Triton X-100, $0.1 \%$ SDS, $1 \mathrm{mM}$ PMSF). The protein concentration was then measured by BCA protein assay. Total $40 \mu \mathrm{g}$ protein extracts were separated on an SDS-polyacrylamide gel and transferred onto a nitrocellulose membrane. The membrane was incubated with primary antibodies: rabbit anti-MYO6 (dilution: 1:500; \#M0691, Sigma), rabbit anti-Cyclin A (dilution: 1:1000; \#18202-1-AP, Proteintech), mouse anti-Cyclin D1 (dilution: 1:1000; \#MD-17-3, Medical \& Biological Laboratories), rabbit anti-p21 (dilution: 1:1000; \#2947, Cell Signaling Technology), rabbit anti-Bax (dilution: 1:500; \#2774, Cell Signaling Technology), rabbit anti-Bcl-2 (dilution: 1:1000; \#2876, Cell Signaling Technology), rabbit anti-cleaved Caspase-3 (dilution: 1:1000; \#25546-1-AP, Proteintech), or mouse anti-GAPDH (dilution: 1:5000; \#Sc-32233, Santa Cruz). Membranes were then washed and incubated with the horseradish peroxidase conjugated goat anti-rabbit IgG (dilution: 1:5000; \#SC-2054, Santa Cruz) or goat anti-mouse IgG (dilution: 1:5000; \#SC-2005, Santa Cruz) for $2 \mathrm{~h}$ at room temperature. Immunoreactivities were developed by using an enhanced chemiluminescence kit (Amersham Life Science, Buckinghamshire, UK). The levels of GAPDH were used as the control.

\subsection{Cell proliferation assay by methylthiazoletetrazolium}

AGS and MGC80-3 cells were infected with Lv-shMYO6(S1), Lv-shMYO6(S2), or Lv-shCon for $96 \mathrm{~h}$, and then all cells were re-seeded in 96-well plates at a density of $2 \times 10^{3}$ cells/well. At indicated time points (1, 2, 3, 4, 5 days), $20 \mu \mathrm{L}$ methylthiazoletetrazolium (MTT) solution $(5 \mathrm{mg} / \mathrm{mL}$; Sigma) was added into the culture medium. After $4 \mathrm{~h}$ incubation, the MTT solution was removed and then 150 $\mu \mathrm{L}$ acidic isopropanol (10\% SDS, $5 \%$ isopropanol and $0.01 \mathrm{~mol} / \mathrm{L} \mathrm{HCl}$ ) was added to each well to dissolve the crystals. The absorbance of each sample was recorded at 595 
nm 10 min later.

\subsection{Colony formation assay}

AGS and MGC80-3 cells were treated with Lv-shMYO6, or Lv-shCon for $96 \mathrm{~h}$, and then both cells were re-seed in 6-well plates (AGS: 500 cells/well, MGC80-3: 400 cells/well). Cells were allowed to form natural colonies in the next 6 days. At the end of time point, cells were washed, fixed by paraformaldehyde and stained with crystal violet, washed by $d_{d H_{2}} \mathrm{O}$, and then photographed with a digital camera. The number of colonies ( $>50$ cells/colony) was counted in both cell lines.

\subsection{Cell cycle analysis}

The cell cycle distribution was analyzed using flow cytometry with PI staining. In brief, both AGS and MGC80-3 cells infected with (Lv-shMYO6, or Lv-shCon) for 3 days were seeded in $6-\mathrm{cm}$ dishes $\left(2 \times 10^{5}\right.$ cells/dish $)$ and cultured at $37^{\circ} \mathrm{C}$ for $40 \mathrm{~h}$. Cells were collected after trypsinization, washed with ice-cold PBS, and fixed with 70\% cold ethanol. Then cells were washed and resuspended in RNase-containing PBS buffer $(100 \mu \mathrm{g} / \mathrm{mL}$ propidium iodide and $10 \mu \mathrm{g} / \mathrm{mL}$ RNase A) on ice. After $30 \mathrm{~min}$, the stained cells were subjected to flow cytometry analysis by a FACs caliber II sorter and Cell Quest FACS system (BD Biosciences, USA). The percentages of cells in three phases (G0/G1, S and G2/M) were statistically analyzed.

\subsection{Apoptosis Assay}

AGS Cells $\left(1 \times 10^{5} /\right.$ well $)$ were collected $48 \mathrm{~h}$ after transfection with Lv-shMYO6, or Lv-shCon and were stained with Annexin V (allophycocyanin [APC] conjugated) and 7-aminoactinomycin $\mathrm{D}$ (7-AAD) according to the manufacturer's instructions (BD Biosciences, Erembodegem, Belgium). Apoptosis was assessed by flow cytometry (BD FACSCalibur).

\subsection{Transwell migration assay}

The cell migration ability was explored via a Transwell chambers with $8.0 \mu \mathrm{m}$ pores 
(Corning Costar, New York, USA). Briefly, MGC80-3 cells $\left(8 \times 10^{4}\right.$ cells/well) were seeded in upper chambers in $200 \mu \mathrm{l}$ serum-free RPMI-1640 medium after lentivirus infection for $96 \mathrm{~h}$. The lower chambers were added into $500 \mu \mathrm{l}$ RPMI-1640 containing $10 \% \mathrm{FBS}$. After cultured for $24 \mathrm{~h}$ at $37^{\circ} \mathrm{C}$, the non-migrated cells on the upper surface were lightly removed by cotton swabs and the migrated cells on the lower surface were fixed with $4 \%$ paraformaldehyde, stained with crystal violet, and counted with five random fields per well.

\subsection{Statistical Analysis}

GraphPad Prism 5 (GraphPad Prism software, USA) was used for the data analysis. All data were expressed as mean \pm standard deviation of three independent experiments. The Student's t-test was used for statistical analysis. A P-value of less than 0.05 was considered significant.

\section{Results}

3.1 MYO6 expression is overexpressed in the gastric cancers and affects patient survival

The differential expression of MYO6 mRNA was analyzed between gastric cancer tissues and normal tissues by Oncomine microarray datasets. We found that expression of MYO6 was significantly elevated in gastric cancer $(n=80, p=7.48 \mathrm{E}-4)$ comparing with the paired normal gastric tissues $(n=80)$ by using Cui Gastric dataset (Fig. 1A). Similarly, MYO6 expression was also higher in gastric cancer ( $\mathrm{n}=15, \mathrm{p}=$ 0.013) than the normal tissues $(\mathrm{n}=12)$ as revealed in Wang Gastric dataset (Fig. 1B). In DErrico Gastric dataset, MYO6 expression was obviously increased in gastric intestinal type adenocarcinoma $(\mathrm{n}=26, \mathrm{p}=1.04 \mathrm{E}-4)$ and gastric mixed adenocarcinoma $(\mathrm{n}=4, \mathrm{p}=0.025)$ in comparison with normal gastric tissues $(\mathrm{n}=31)$ (Fig. 1C). Furthermore, MYO6 was upregulated in gastric intestinal type adenocarcinoma $(n=54, p=0.002)$ comparing with normal tissues $(n=22)$ in another independent dataset Chen Gastric (Fig. 1D). What's more, we analyzed the correlation between MYO6 mRNA expression levels and overall survival (OS) in 
gastric cancer patients using the Kaplan-Meier plotter online software based on a public database. We found that the OS of patients with low expression of MYO6 was remarkably longer than those patients with high expression $(\mathrm{HR}=0.75,95 \% \mathrm{CI}=$ 0.63-0.89, $p<0.01$, Fig. 2). Taken together, our findings indicated that high expression of MYO6 has the significant clinical importance and acts as a prognostic marker for gastric cancer patients.

\subsection{Knockdown of MYO6 by shRNA lentiviral system in gastric cancer cells}

To investigate the role of MYO6 in human gastric cancer, the expression levels of MYO6 were analyzed in three different gastric cancer cell lines by qRT-PCR and western blot. As shown in Fig. 3A and B, MYO6 was expressed in all three cell lines. Both the mRNA and protein levels of MYO6 were lowest in SGC-7901 cells among these cell lines. Therefore, AGS and MGC80-3 cells were selected as cellular models for further loss-of-function investigation. For MYO6 knockdown, a lentivirus mediated RNAi system was applied. AGS, MGC80-3 and SGC-7901 cells were subjected to two different treatments (Lv-shCon and Lv-shMYO6 (S1)) or three different treatments (Lv-shCon, Lv-shMYO6 (S1) and Lv-shMYO6(S2)), respectively. To identify the infection efficiency, fluorescence microscopy was employed. More than $80 \%$ of cells were successfully infected with Lv-shMYO6 (S1), Lv-shMYO6 (S2), or Lv-shCon in both AGS and MGC80-3 cell lines, as revealed by GFP fluorescence (Fig. 3C, D). To confirm the silencing effect of Lv-shMYO6 on endogenous MYO6 expression, qRT-PCR and western blot analysis were performed in both AGS and MGC80-3 cell lines. As shown in Fig. 3E-G, the mRNA expression levels of MYO6 were significantly down regulated in AGS and MGC80-3 cells after MYO6 knockdown. Moreover, the protein levels of MYO6 were also markedly decreased in both AGS and MGC80-3 cells after MYO6 knockdown, indicating that the efficient silencing of MYO6 expression in both cell lines (Fig. 3H-J). Similarly, Lv-shCon, Lv-shMYO6 (S1), and Lv-shMYO6 (S2) also efficiently transduced into SGC-7901 cells (Fig. S1A). The protein levels of MYO6 were significantly decreased in Lv-shMYO6 (S1) and Lv-shMYO6 (S2) groups (Fig. S1B). 


\subsection{MYO6 knockdown induced proliferation inhibition in gastric cancer cells}

The potential role of MYO6 in gastric cancer cell proliferation was determined by MTT assay. Our data revealed that the proliferation of both AGS and MGC80-3 cells was remarkably repressed after Lv-shMYO6 infection. On the 5th day of infection, the cell proliferation rates of Lv-shMYO6(S1) groups were significantly decreased by 49.7\% and 79.7\% in AGS and MGC80-3 cells compared to Lv-shCon groups $(p<$ 0.001, Fig. 4A, B). Similarly, a 76\% reduction in cell proliferation of Lv-shMYO6(S2) groups was also observed in MGC80-3 cells $(p<0.001$, Fig. 4C). However, the proliferation rate of SGC-7901 cells was no significant difference between MYO6 knockdown groups (Lv-shMYO6(S1) and Lv-shMYO6(S2)) and Lv-shCon group (Fig. S1C), which may be due to the low endogenous expression of MYO6 in SGC-7901 cells. The above results showed that off-target effects of siRNAs targeted MYO6 gene were avoided and we would choose Lv-shMYO6(S1)-transduced cells A549 and MGC80-3 to perform the subsequent experiments.

\subsection{MYO6 knockdown resulted in impaired colony formation capacity}

To study the long-term effect of MYO6 on cell growth, we studied the colony formation capacity in both AGS and MGC80-3 cell lines. Cells in two groups (Lv-shCon, Lv-shMYO6) were allowed to form natural colonies. At indicated time point, colonies formed in both cell lines were stained and observed. The number of colonies and the size of each colony were both significantly decreased in AGS and MGC80-3 cells after MYO6 knockdown (Fig. 4D, E). As revealed in Fig. 4F and G, the number of colonies in Lv-shMYO6 groups (AGS: $18 \pm 1$, MGC80-3: $33 \pm 4$ ) was reduced by over 80\%, as compared to Lv-shCon (AGS: $111 \pm 3$, MGC80-3: $165 \pm 10$ ). Collectively, knockdown of MYO6 remarkably suppressed the proliferation and colony formation capacity of gastric cancer cells.

\subsection{MYO6 knockdown induced cell cycle arrest in gastric cancer cells}

To further illustrate how MYO6 mediates cell growth, we performed a cell cycle 
analysis in AGS and MGC80-3 cells by flow cytometry (Fig. 5A and B). As shown in Fig. 5C, the cell percentage of G0/G1 phase in Lv-shMYO6 group was significantly increased $(55.49 \pm 0.37 \%$ vs. $40.56 \pm 0.52 \%, p<0.001)$, while the cell percentage of S phase was decreased $(20.05 \pm 0.22 \%$ vs. $34.15 \pm 1.06 \%, p<0.01)$ compared with Lv-shCon group in AGS cells. Similarly, knockdown of MYO6 increased the proportion of cells in G0/G1 phase $(55.37 \pm 1.81 \%$ vs. $48.34 \pm 0.24 \%, p<0.05)$ and $\mathrm{G} 2 / \mathrm{M}$ phase $(28.36 \pm 0.88 \%$ vs. $22.15 \pm 0.61 \%, p<0.01)$, decreased in S phase $(16.26 \pm 1.33 \%$ vs. $29.51 \pm 0.59 \%, p<0.001)$ in MGC80-3 cells (Fig. 5D). These results strongly indicated that knockdown of MYO6 blocked cell cycle progression of gastric cancer cells.

\subsection{MYO6 knockdown altered the expression of cell cycle regulatory}

Accumulating evidence shows that the progression of tumorigenesis involves abnormalities in the expression of cyclins and related genes. Cyclin D1 and cyclin A are strongly activated during G1 phase and transition from G1 to the S phase of the cell cycle in the gastric cancer cells, respectively (Han et al., 1999; Li et al., 2002; Ioachim, 2008). In addition, these progressive factors can be inhibited by blockers, such as p21. The qRT-PCR and western blot analysis revealed that MYO6 knockdown led to strong suppression of progressive factors cyclin A and cyclin D1 activation and largely released cyclin-dependent kinase inhibitor p21 in MGC80-3 cells. Therefore, MYO6 provides a powerful potential bio-marker for gastric cancer diagnosis (Fig. 5E, F).

\subsection{MYO6 knockdown caused cell apoptosis}

In order to determine the effect of depletion of MYO6, apoptosis assay with staining with 7-aminoactinomycin D (7-ADD) and Annexin V was performed on AGS and MGC80-3 cells. This staining combination enables viable, apoptotic, and necrotic cells to be distinguished. As expected, knockdown of MYO6 induced apoptosis of AGS and MGC80-3 cells (Fig. 6A, C). As shown in Fig. 6B, Lv-shMYO6-infected AGS cells promotes more early and late apoptosis $(9.63 \% \pm 0.36 \%, p<0.001,5.23 \%$ 
$\pm 0.32 \%, p<0.01$, respectively), compared with control cells $(5.91 \% \pm 0.21 \%, 3.48 \%$ $\pm 0.15 \%$, respectively). In total, an approximately 2 -fold increase in apoptotic populations in Lv-shMYO6 compared to Lv-shCon group in AGS cells. Similar results were obtained in MGC80-3 cells. Specifically, the percentage of apoptotic cells in Lv-shMYO6 group was increased more than triple (early apoptosis: $14.70 \pm$ $0.46 \%$ vs. $4.47 \pm 0.72 \%$, late apoptosis: $8.75 \pm 1.84 \%$ vs. $2.67 \pm 0.50 \%, p<0.05, p<$ 0.001) in comparison with shCon group in MGC80-3 cells (Fig. 6D). Statistical analysis reveals that the lack of MYO6 results in apoptotic cell death.

To further confirm the influence of MYO6 silencing on cell apoptosis, we performed western blotting assay to detect the expression of apoptosis-related proteins including Bax, Bcl-2 and cleaved Caspase-3 in MGC80-3 cells. We found that depletion of MYO6 increased the expression of Bax and cleaved Caspase-3, and decreased the expression of Bcl-2 protein (Fig. 6E), suggesting that the involvement of MYO6 silencing on cell apoptosis may be partly through activating the expression of Bax and cleaved Caspase-3, and controlling Bcl-2 expression.

\subsection{MYO6 knockdown inhibited migration of MGC80-3 cells}

To explore whether MYO6 knockdown could affect gastric cancer cell migration, we further carried out the transwell assay in MGC80-3 cells. As shown in Fig. 7A and $\mathrm{B}$, the migration number of MGC80-3 cells was significantly decreased in the Lv-shMYO6 group $(9.3 \pm 0.6$ vs. $209.4 \pm 2.7, p<0.001)$ in comparison with the Lv-shCon group, suggesting that MYO6 gene may be also play an important role in gastric cancer cell migration.

\section{Discussion}

Gastric cancer is one of the leading causes of cancer-related death worldwide. Recently, a great number of cancer-related genes during gastric cancer progression have been identified. Myosins, a group of ATP-dependent motor proteins, are reported to be responsible for actin-based motility, trafficking, intracellular transport, and possible regulation of signal transduction in non-muscle cells (Wu et al., 2000; Berg 
et al., 2001; Dunn et al., 2006). MYO6 was shown to function in iris development, maintaining epithelial barrier function and mammalian auditory systems (Liao et al., 2013; Samuels et al., 2013; Williams et al., 2013). Besides, MYO6 has also been shown to play a critical role in cancer cell migration including ovary cancers and prostate cancers (Yoshida et al., 2004; Dunn et al., 2006; Wei et al., 2008).

Currently, more and more studies have reported that MYO6 mediates cell viability via cell-cycle arrest and induces apoptosis in various tumor cells including colorectal cancer (You et al., 2016), glioma (Xu et al., 2016), breast cancer (Wang et al., 2015), and lung cancer (Yu et al., 2015). Moreover, MYO6 as a potential urinary biomarker could enhance prostate cancer detection (Mengual et al., 2015). However, so far, there is no study focused on the role of MYO6 in gastric cancer. In the present study, Herein, we first showed that MYO6 plays an underlying oncogenic role in human gastric cancer. In our study, Oncomine database and Online Kaplan-Meier plotter analysis proved that MYO6 is over expressed and predicts a poor prognosis in gastric cancer patients. We also discovered MYO6 knockdown could remarkably inhibit the growth and proliferation of two typical human gastric cancer cells AGS and MGC80-3 via MTT and colony formation assays. Moreover, cell cycle analysis revealed that the underlying mechanism of MYO6 regulation cell growth was involved in the cell cycle arrest at G0/G1 phase after MYO6 inhibition. Remarkably, knockdown of MYO6 resulted in the increase proportion of cells in G2/M phase in MGC80-3 cells but not in AGS cells. The difference in G2/M phase in AGS and MGC80-3 cells may be due to the different cell sources that AGS cells is from fragments of a tumor resected from a patient who had received no prior therapy (Barranco et al., 1983), while MGC80-3 cells is from a 53-year-old male patients with primary poorly differentiated gastric mucous adenocarcinoma (Liu et al., 2002). Furthermore, apoptosis assay suggest that MYO6 inhibition early and late apoptosis in AGS and MGC80-3 cells. Additionally, depletion of MYO6 could also suppress the migration of MGC80-3 cells in this study. Therefore, the high expression of MYO6 gene may be mainly correlated with gastric cancer cell proliferation and migration, and play a crucial role in the development and progression of gastric cancer. 
Cell division is one of the most tightly controlled steps of the cell cycle. During cell division, the acto-myosin contraction is precisely regulated to ensure that cytokinesis takes place properly (Lafaurie-Janvore, 2013). Cyclin D1 and cyclin A are two cell-cycle-related progressive molecules, which are strongly activated during G1 phase and transition from G1 to the S phase of the cell cycle, respectively (Baldin et al., 1993; Henglein et al., 1994). Moreover, previous studies reported that p21 was an effective cyclin-dependent kinase inhibitor, and could act as a regulator of cell cycle progression at G1 and S phase (Gartel and Radhakrishnan, 2005). The qRT-PCR and western blot analysis revealed knockdown of MYO6 significantly inhibited cell-cycle-related progressive molecules cyclin A and cyclin D1 expression, and promoted cell-cycle-related blocker p21 expression. In consistent, myosin superfamily members also play roles in cell cycle control. Non-muscle myosin II (NMII) has pivotal roles in cell division, migration and differentiation, as inhibition of NMII leads to G0/G1 arrest of Wharton's jelly-derived mesenchymal stromal cells (Sharma et al., 2013). MYOD has been reported to induce cell cycle arrest during skeletal muscle differentiation by increasing the expression of p21 (Halevy et al., 1995). In Rat 2 cells, MYO6 knockdown altered cell cycle distribution (Cameron et al., 2013). Furthermore, western blot analysis showed that knockdown of MYO6 obviously affected the expression of Bax, Bcl-2 and cleaved Caspase-3. Bcl-2 is the most representative of anti-apoptotic gene in the Bcl-2 family, which could regulate the release of apoptosis-related factors (Rosse et al., 1998). Bax, as a pro-apoptotic protein member of Bcl-2 family, could counter the death repressor activity of Bcl-2 (Oltvai et al., 1993). And Caspase 3, a pivotal regulator of apoptosis, is essential for apoptotic chromatin condensation and DNA fragmentation (Porter and Janicke, 1999). Taken together, MYO6 could modulate gastric cancer cell growth via cell cycle and apoptosis regulations, and thus may serve as a potential therapeutic target in human gastric cancer.

In conclusion, our study presents a basic conception that MYO6 mediates gastric cancer cell survival through enhancing cell growth and inhibiting apoptosis. Further investigation is still needed to reveal the exactly regulatory mechanism of MYO6 to 
provide more direct evidence that MYO6 may act as a novel candidate therapeutic target against gastric cancer.

\section{Acknowledgments}

The authors are thankful for the financial support from the Anhui province national science foundation of China (1508085MH146)

\section{References}

Baldin, V., et al., 1993. Cyclin D1 is a nuclear protein required for cell cycle progression in G1. Genes Dev 7, 812-821.

Barranco, S.C., et al., 1983. Establishment and characterization of an in vitro model system for human adenocarcinoma of the stomach. Cancer Res 43, 1703-1709.

Berg, J.S., et al., 2001. A millennial myosin census. Mol Biol Cell 12, 780-794.

Buss, F., et al., 2004. Myosin VI: cellular functions and motor properties. Annu Rev Cell Dev Biol 20, 649-676.

Cameron, R.S., et al., 2013. Myosin 16 levels fluctuate during the cell cycle and are downregulated in response to DNA replication stress. Cytoskeleton (Hoboken) $70,328-348$.

Chen, X., et al., 2003. Variation in gene expression patterns in human gastric cancers. Mol Biol Cell 14, 3208-3215.

Cui, J., et al., 2011. An integrated transcriptomic and computational analysis for biomarker identification in gastric cancer. Nucleic Acids Res 39, 1197-1207.

D'Errico, M., et al., 2009. Genome-wide expression profile of sporadic gastric cancers with microsatellite instability. Eur J Cancer 45, 461-469.

Dunn, T.A., et al., 2006. A novel role of myosin VI in human prostate cancer. Am J Pathol 169, 1843-1854.

Gartel, A.L. and Radhakrishnan, S.K., 2005. Lost in transcription: p21 repression, mechanisms, and consequences. Cancer Res 65, 3980-3985.

Gyorffy, B., et al., 2010. An online survival analysis tool to rapidly assess the effect of 
22,277 genes on breast cancer prognosis using microarray data of 1,809 patients. Breast Cancer Res Treat 123, 725-731.

Halevy, O., et al., 1995. Correlation of terminal cell cycle arrest of skeletal muscle with induction of p21 by MyoD. Science 267, 1018-1021.

Han, S., et al., 1999. Expression of p27Kip1 and cyclin D1 proteins is inversely correlated and is associated with poor clinical outcome in human gastric cancer. J Surg Oncol 71, 147-154.

Henglein, B., et al., 1994. Structure and cell cycle-regulated transcription of the human cyclin A gene. Proc Natl Acad Sci U S A 91, 5490-5494.

Ioachim, E., 2008. Expression patterns of cyclins D1, E and cyclin-dependent kinase inhibitors p21waf1/cip1, p27kip1 in colorectal carcinoma: correlation with other cell cycle regulators (pRb, p53 and Ki-67 and PCNA) and clinicopathological features. Int J Clin Pract 62, 1736-1743.

Kellerman, K.A. and Miller, K.G., 1992. An unconventional myosin heavy chain gene from Drosophila melanogaster. J Cell Biol 119, 823-834.

Krendel, M. and Mooseker, M.S., 2005. Myosins: tails (and heads) of functional diversity. Physiology (Bethesda) 20, 239-251.

Lafaurie-Janvore, J., 2013. [Temporal regulation of abscission, the last step of cell division]. Biol Aujourdhui 207, 133-148.

Li, J.Q., et al., 2002. Cyclin A correlates with carcinogenesis and metastasis, and p27(kip1) correlates with lymphatic invasion, in colorectal neoplasms. Hum Pathol 33, 1006-1015.

Liao, Y.W., et al., 2013. Myosin VI contributes to maintaining epithelial barrier function. J Biomed Sci 20, 68.

Liu, S., et al., 2002. Induction of apoptosis by TPA and VP-16 is through translocation of TR3. World J Gastroenterol 8, 446-450.

Mengual, L., et al., 2015. Using gene expression from urine sediment to diagnose prostate cancer: development of a new multiplex mRNA urine test and validation of current biomarkers. BMC Cancer 16, 76.

O'Connell, C.B., et al., 2007. Myosin at work: motor adaptations for a variety of 
cellular functions. Biochim Biophys Acta 1773, 615-630.

Olson, M.F. and Sahai, E., 2009. The actin cytoskeleton in cancer cell motility. Clin Exp Metastasis 26, 273-287.

Oltvai, Z.N., et al., 1993. Bcl-2 heterodimerizes in vivo with a conserved homolog, Bax, that accelerates programmed cell death. Cell 74, 609-619.

Pollard, T.D. and Korn, E.D., 1973. Acanthamoeba myosin. I. Isolation from Acanthamoeba castellanii of an enzyme similar to muscle myosin. J Biol Chem 248, 4682-4690.

Porter, A.G. and Janicke, R.U., 1999. Emerging roles of caspase-3 in apoptosis. Cell Death Differ 6, 99-104.

Puri, C., et al., 2010. Overexpression of myosin VI in prostate cancer cells enhances PSA and VEGF secretion, but has no effect on endocytosis. Oncogene 29, 188-200.

Rhodes, D.R., et al., 2004. ONCOMINE: a cancer microarray database and integrated data-mining platform. Neoplasia 6, 1-6.

Rosse, T., et al., 1998. Bcl-2 prolongs cell survival after Bax-induced release of cytochrome c. Nature 391, 496-499.

Samuels, I.S., et al., 2013. Myosin 6 is required for iris development and normal function of the outer retina. Invest Ophthalmol Vis Sci 54, 7223-7233.

Sharma, T., et al., 2013. Inhibition of non-muscle myosin II leads to G/G arrest of Wharton's jelly-derived mesenchymal stromal cells. Cytotherapy.

Wang, H., et al., 2015. Lentivirus-Mediated Knockdown of Myosin VI Inhibits Cell Proliferation of Breast Cancer Cell. Cancer Biother Radiopharm 30, 330-335.

Wang, Q., et al., 2012. Upregulated INHBA expression is associated with poor survival in gastric cancer. Med Oncol 29, 77-83.

Wei, S., et al., 2008. GOLPH2 and MYO6: putative prostate cancer markers localized to the Golgi apparatus. Prostate 68, 1387-1395.

Wells, A.L., et al., 1999. Myosin VI is an actin-based motor that moves backwards. Nature 401, 505-508.

Williams, L.H., et al., 2013. Characterization of a novel ENU-generated myosin VI 
mutant mouse strain with congenital deafness and vestibular dysfunction. Hear Res 299, 53-62.

Wu, X., et al., 2000. Functions of unconventional myosins. Curr Opin Cell Biol 12, 42-51.

$\mathrm{Xu}$, R., et al., 2016. Myosin VI contributes to malignant proliferation of human glioma cells. Korean J Physiol Pharmacol 20, 139-145.

Yoshida, H., et al., 2004. Lessons from border cell migration in the Drosophila ovary: A role for myosin VI in dissemination of human ovarian cancer. Proc Natl Acad Sci U S A 101, 8144-8149.

You, W., et al., 2016. Downregulation of myosin VI reduced cell growth and increased apoptosis in human colorectal cancer. Acta Biochim Biophys Sin (Shanghai) 48, 430-436.

Yu, H., et al., 2015. Lentivirus-Mediated Silencing of Myosin VI Inhibits Proliferation and Cell Cycle Progression in Human Lung Cancer Cells. Chem Biol Drug Des 86, 606-613.

\section{Figure legends}

Fig. 1. MYO6 mRNA expression in human gastric cancers via the Oncomine database. (A, B) Differential expression of MYO6 in the normal and human gastric cancer tissues was revealed by Cui Gastric dataset and Wang Gastric dataset, respectively. (C) MYO6 mRNA expression analysis in the normal gastric, gastric intestinal type adenocarcinoma and gastric mixed adenocarcinoma tissues in DErrico Gastric dataset was shown. (D) MYO6 mRNA expression in the normal and gastric intestinal type adenocarcinoma specimens in Chen Gastric dataset was different.

Fig. 2. The correlation of MYO6 mRNA expression with overall survival in gastric cancer patients. The Kaplan-Meier plots analysis for overall survival in gastric cancer patients according to the expression levels of MYO6. HR: hazard ratio; CI: confidence interval; $p$ value: log-rank test.

Fig. 3. Knockdown of MYO6 expression by RNA interference. (A) QRT-PCR and (B) western blot were applied to analyze the expression levels of MYO6 in SGC-7901, 
MGC80-3 and AGS cells. (C) The infected efficiency of Lv-shCon and Lv-shMYO6(S1) in AGS cells were observed by fluorescence microscopy. (D) The infected efficiency of Lv-shCon, Lv-shMYO6(S1) and Lv-shMYO6(S2) in MGC80-3 cells were observed by fluorescence microscopy. (E) The mRNA levels of MYO6 in AGS cells following Lv-shMYO6(S1) infection were analyzed by qRT-PCR. (F, G) The mRNA levels of MYO6 in MGC80-3 cells following Lv-shMYO6(S1)/(S2) infection were analyzed by qRT-PCR. (H) The protein levels of MYO6 in AGS cells following Lv-shMYO6(S1) infection were analyzed by western blot. (I, J) The protein levels of MYO6 in MGC80-3 cells following Lv-shMYO6(S1)/(S2) infection were analyzed by western blot. $\beta$-actin gene and GAPDH protein were used as internal controls. Scale bar: $100 \mu \mathrm{m}$; ** $p<0.01$, ***p $<0.001$, compared to LV-shCon.

Fig. 4. MYO6 knockdown suppressed gastric cancer cell proliferation. (A) The proliferation of AGS cells with two different treatments Lv-shCon and Lv-shMYO6(S1) was determined by MTT assay. (B, C) The proliferation of MGC80-3 cells with different treatments Lv-shCon and Lv-shMYO6(S1)/(S2) was determined by MTT assay, respectively. (D, E) Representative images of colonies in AGS and MGC80-3 cells under light microscopy and fluorescence microscopy. (F, G) Statistical analysis of the number of colonies in AGS and MGC80-3 cells with crystal violet staining presented. Scale bar: $250 \mu \mathrm{m}$; *** $p<0.001$, compared to Lv-shCon.

Fig. 5. MYO6 knockdown induced cell cycle arrest. (A, B) Cell cycle distribution of AGS and MGC80-3 cells was analyzed by flow cytometry. The X-axis represents DNA content and the Y-axis represents the number of cells. (C, D) Statistical analysis revealed the percentage of AGS cells and MGC80-3 cells in each phase of the cell cycle. (E) QRT-PCR and (F) western blot were applied to analyze the expression levels of cyclin A, cyclin D1 and p21 in Lv-shCon- or Lv-shMYO6-infected MGC80-3 cells. $\beta$-actin gene and GAPDH protein were used as internal controls. ${ }^{*} p<$ $0.05, * * p<0.01, * * * p<0.001$, compared to Lv-shCon.

Fig. 6. MYO6 knockdown induced cell apoptosis in AGS and MGC80-3 cells. (A, C) Apoptosis cells were assessed by Annexin V/7-ADD staining and then were subjected to flow cytometry for analysis. Representative images showing Annexin V/7-ADD 
staining results in AGS and MGC80-3 cells. (B, D) Quantification of the percentage of cells in early and late apoptosis in AGS and MGC80-3 cells, respectively. (E) western blot was applied to analyze the expression levels of Bax, Bcl-2 and cleaved Caspase-3 in Lv-shCon- or Lv-shMYO6-infected MGC80-3 cells. GAPDH protein were used as internal controls. $* p<0.05, * * p<0.01, * * * p<0.001$, compared to Lv-shCon.

Fig. 7. MYO6 knockdown inhibited migration of MGC80-3 cells. (A) Representative images of cell migration in MGC80-3 cells under light microscopy. (B) Statistical analysis of the number of migration cells with crystal violet staining presented. Scale bar: $100 \mu \mathrm{m} ; * * * p<0.001$, compared to Lv-shCon. 
A

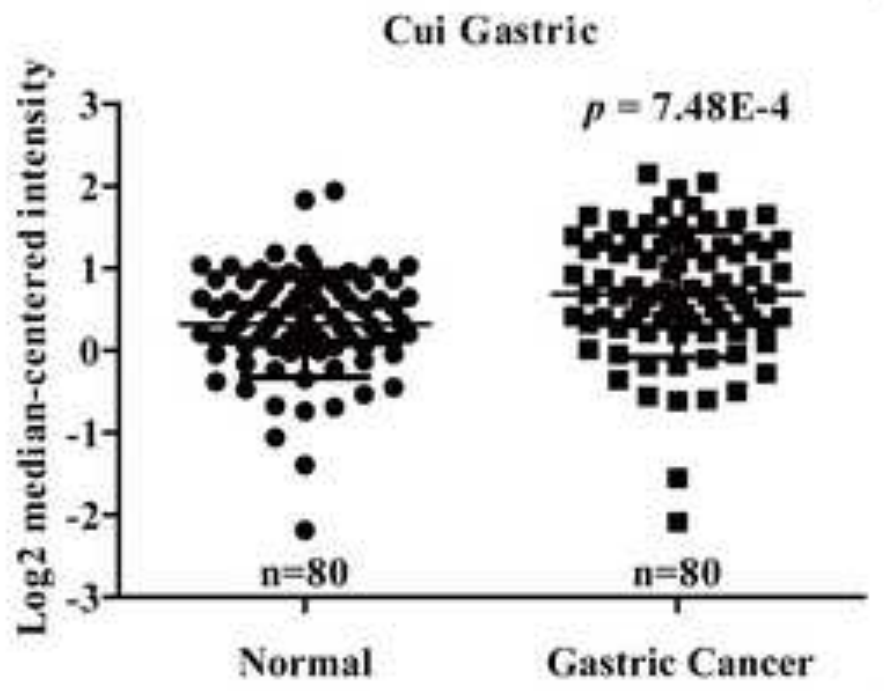

C

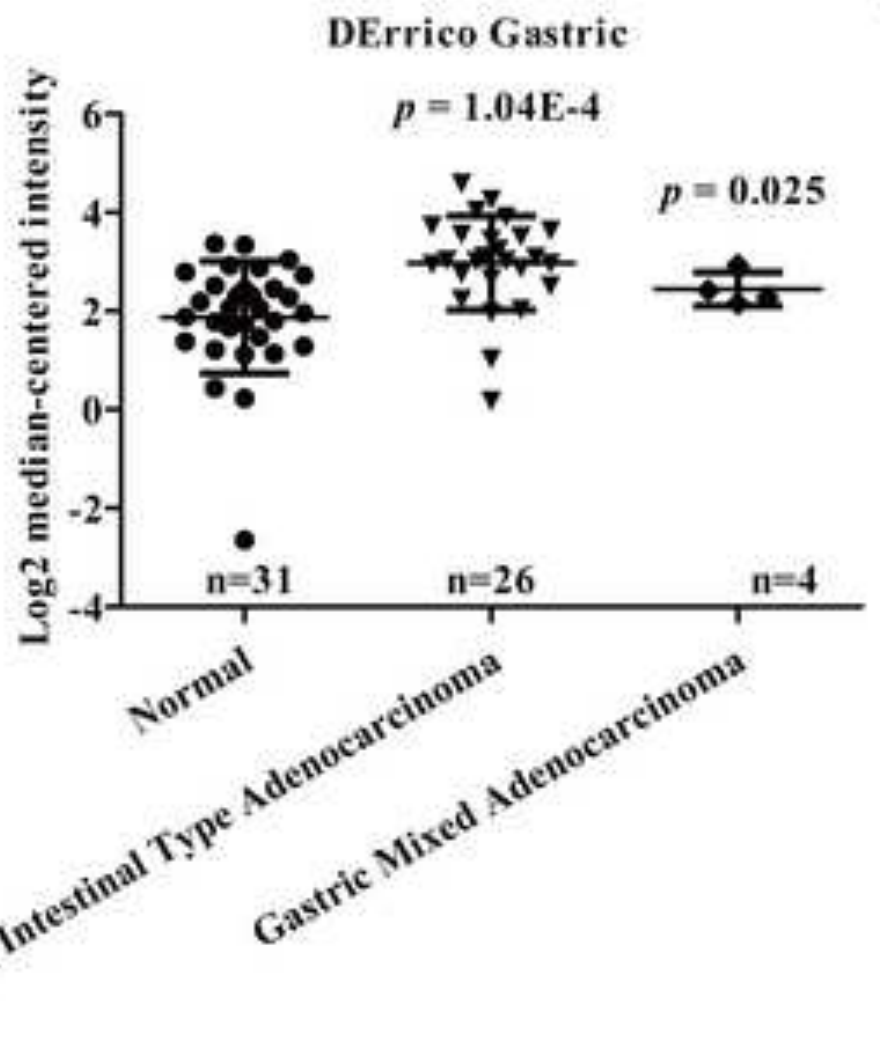

B

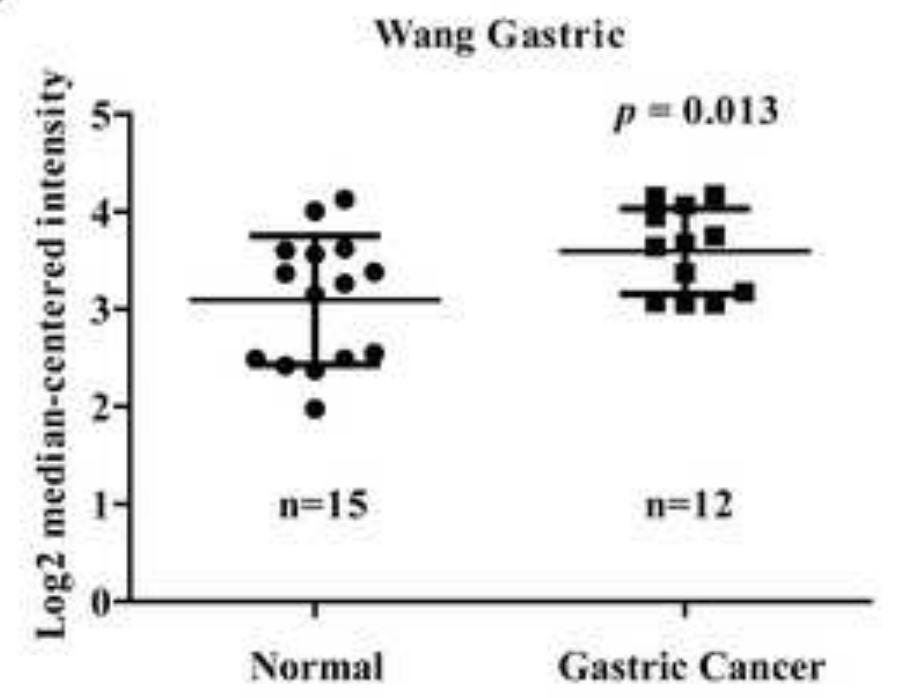

D

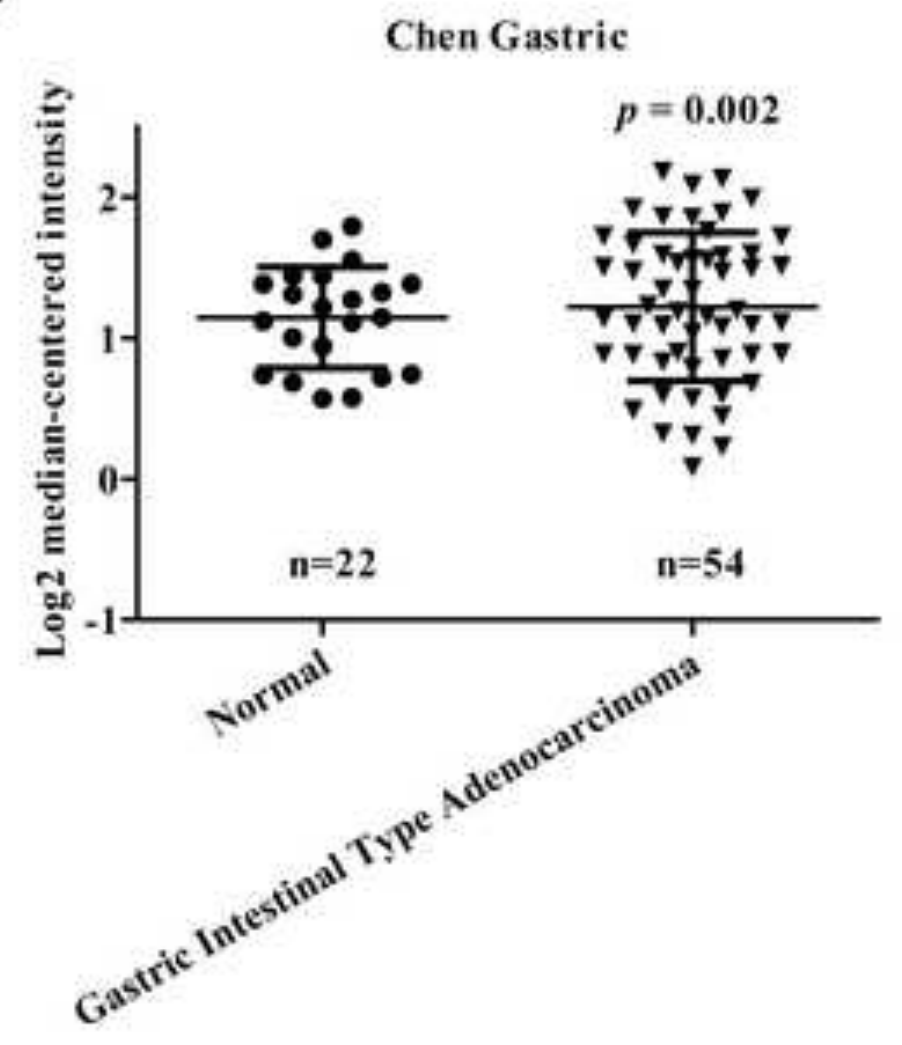




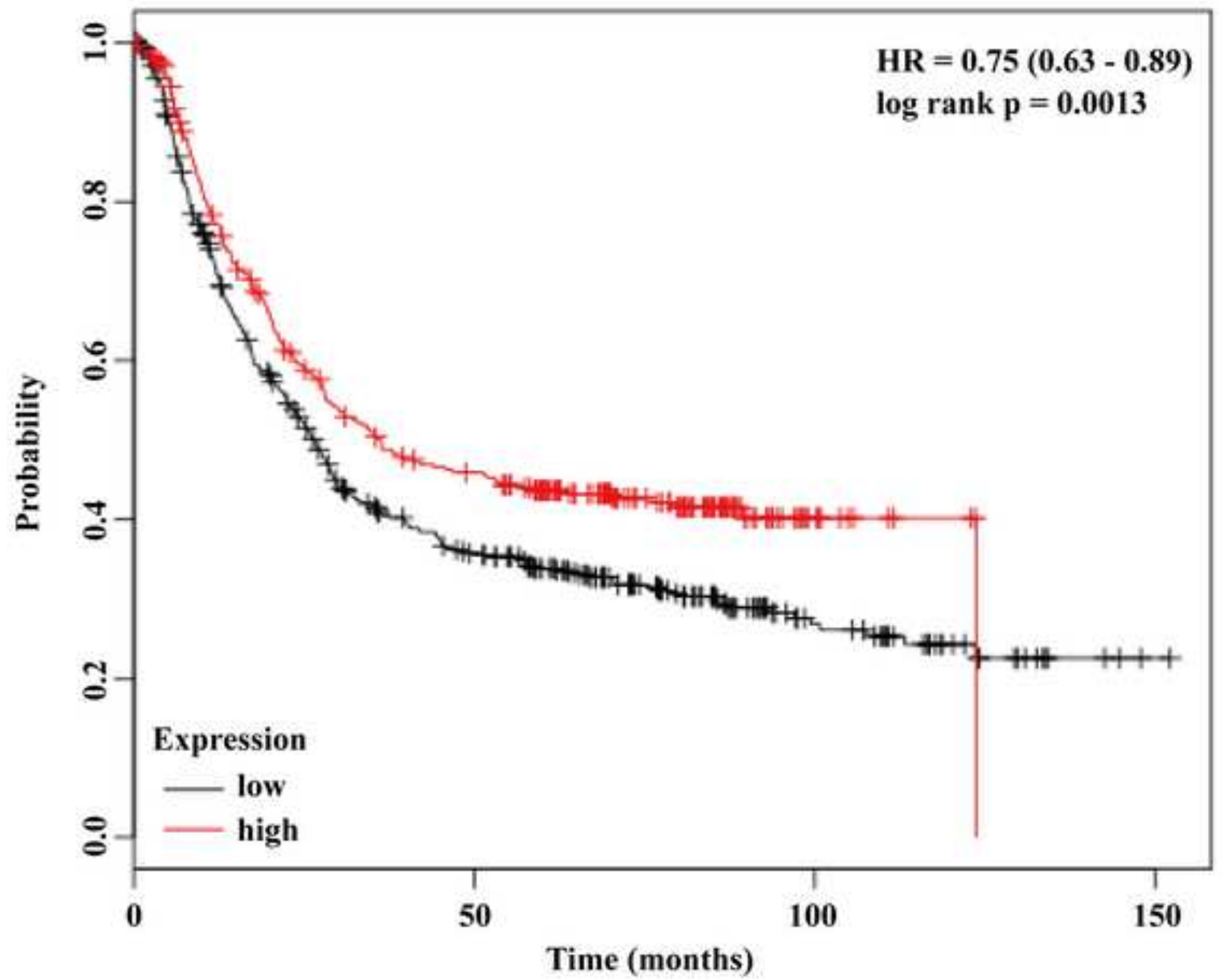

Number at risk

low $\quad 529$

156

36

1

high 347

142

12

0 
A

MYO6

B
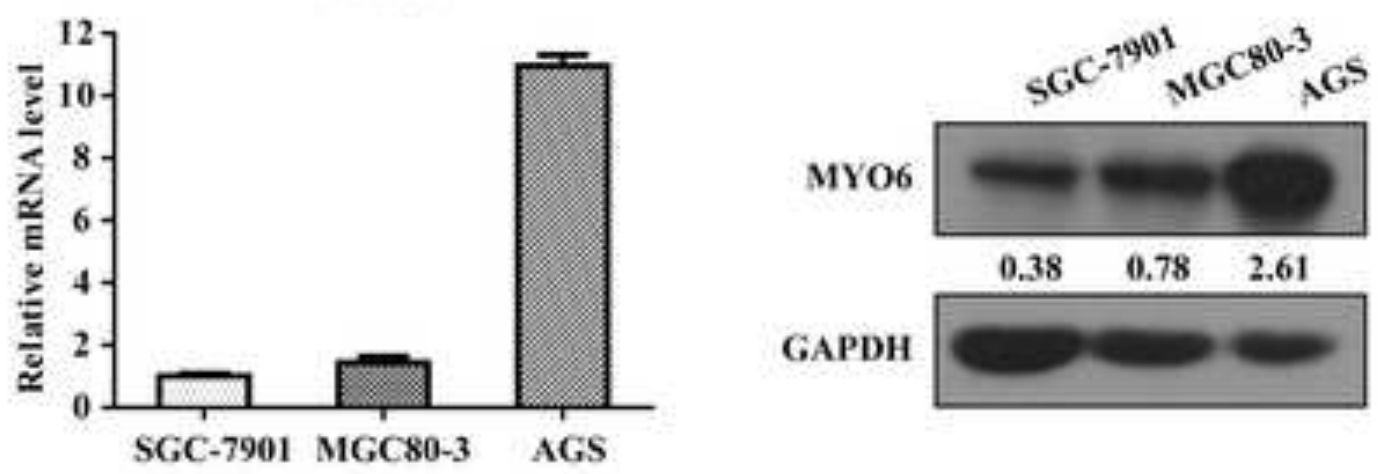

C

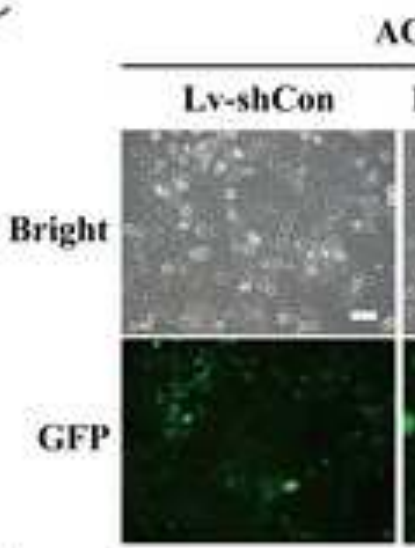

AGS

D

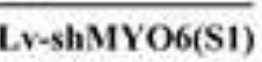

E
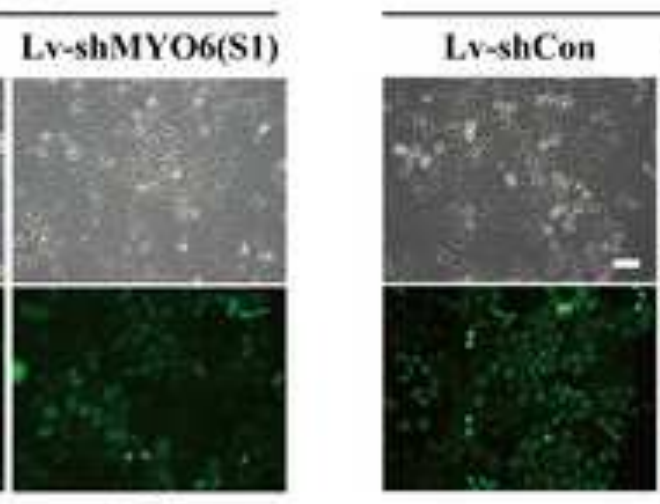

MGC80-3

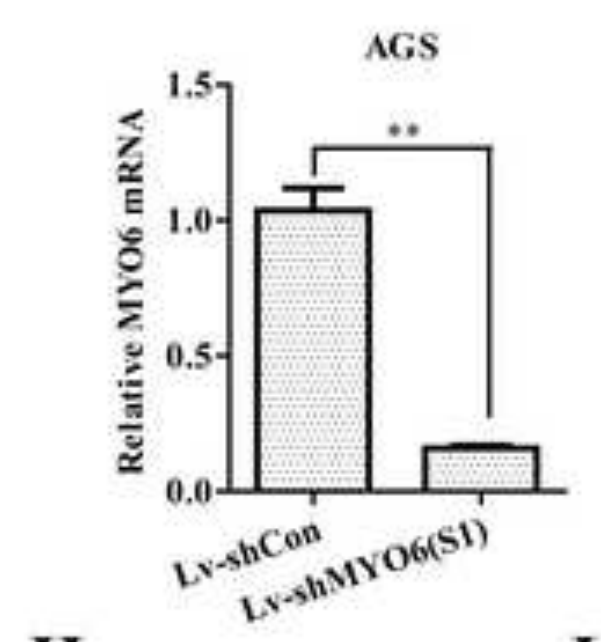

H

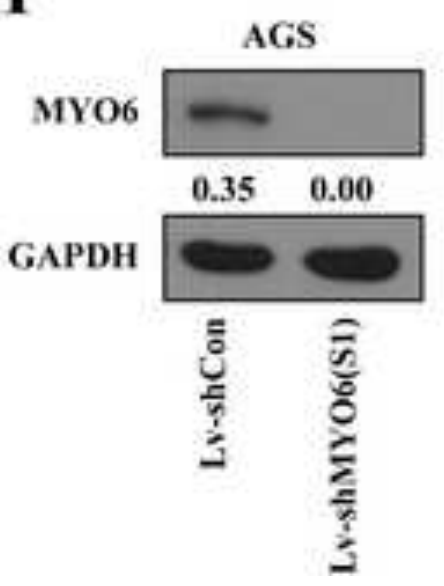

Lv-shMYO6(S1) Lv-shMYO6(S2)

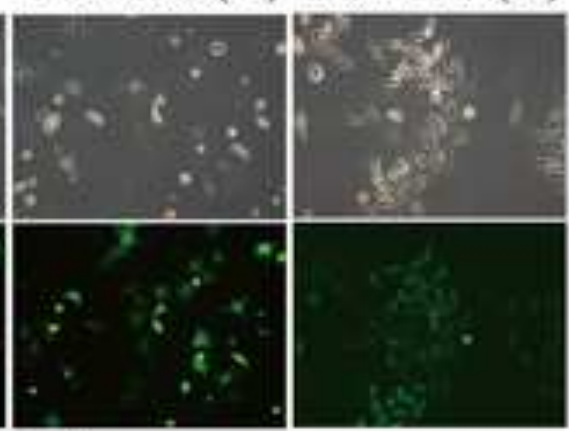

G
MGC80-3

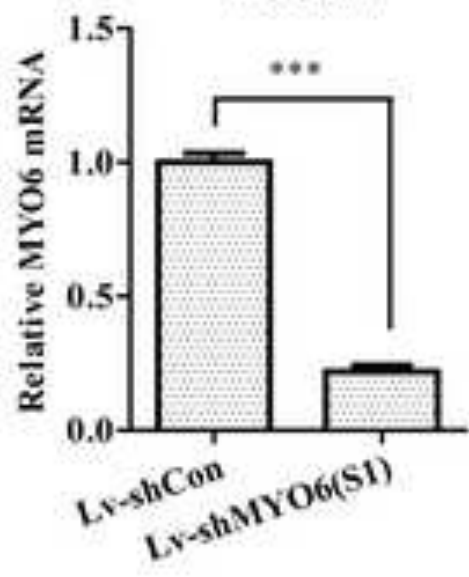

MGC80-3

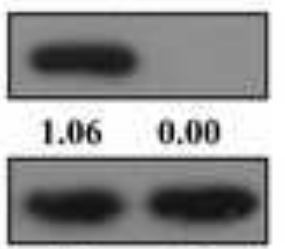

产
MGC80-3

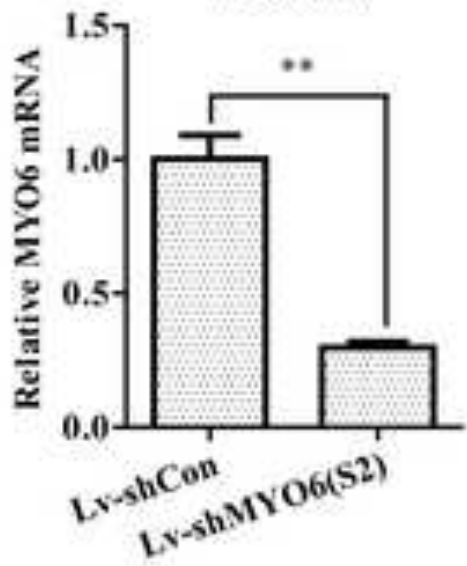

MGC80-3

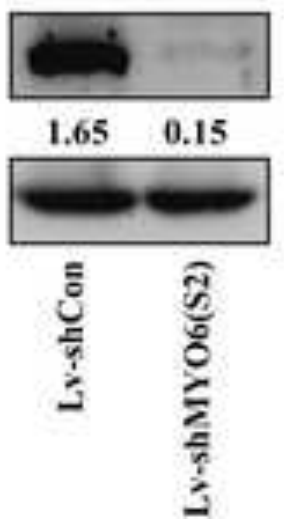


A

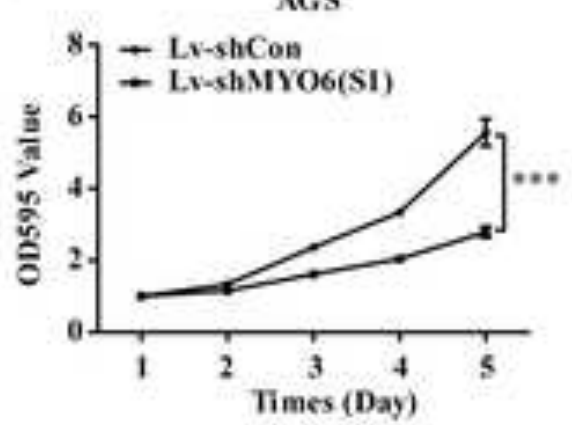

D

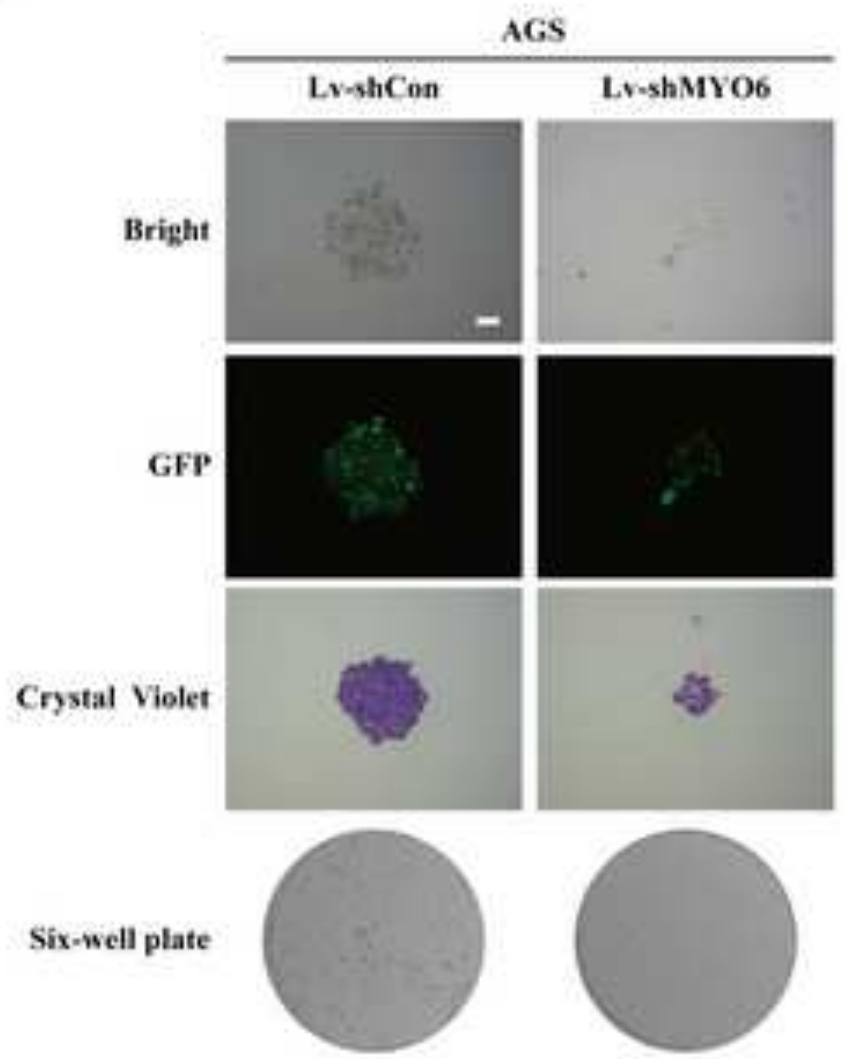

F

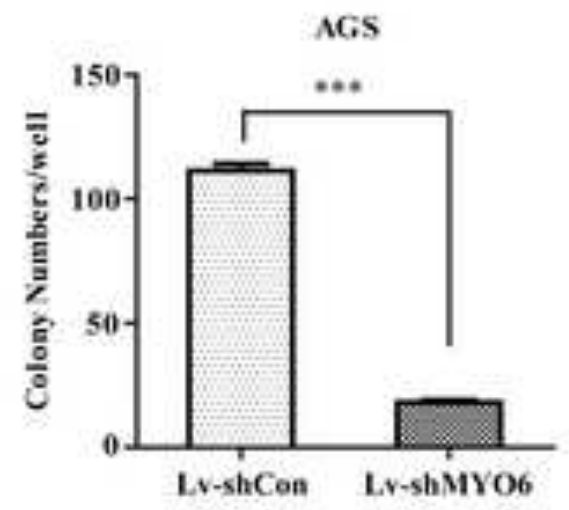

MGC80-3

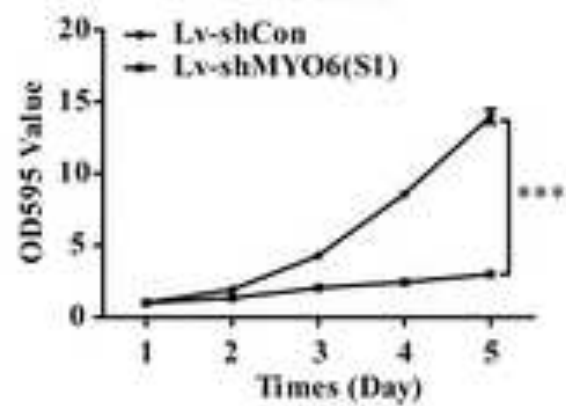

E

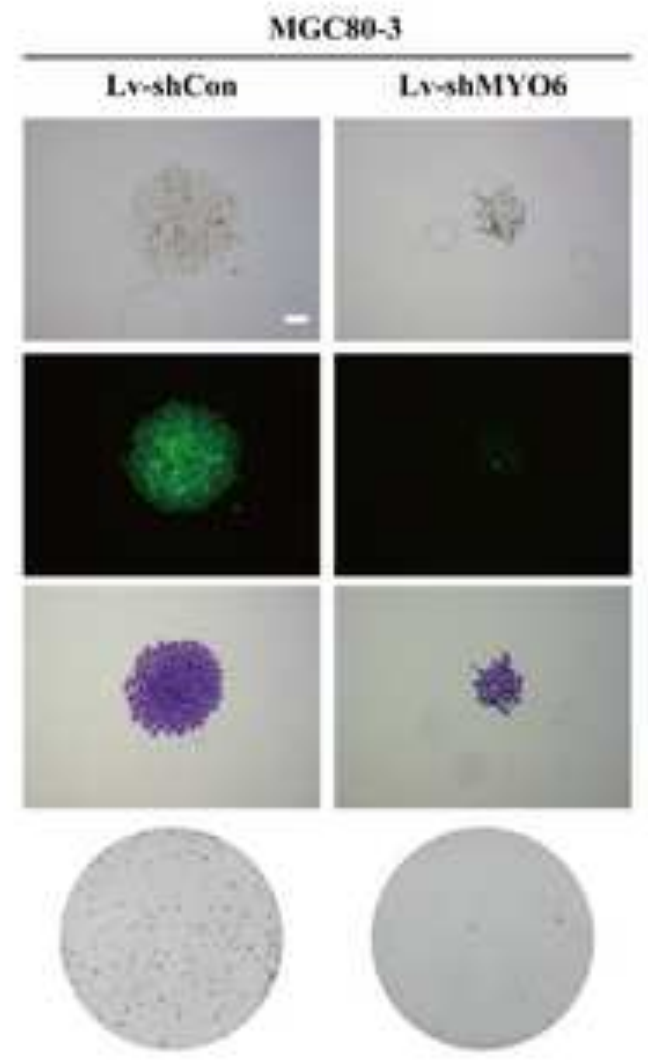

G
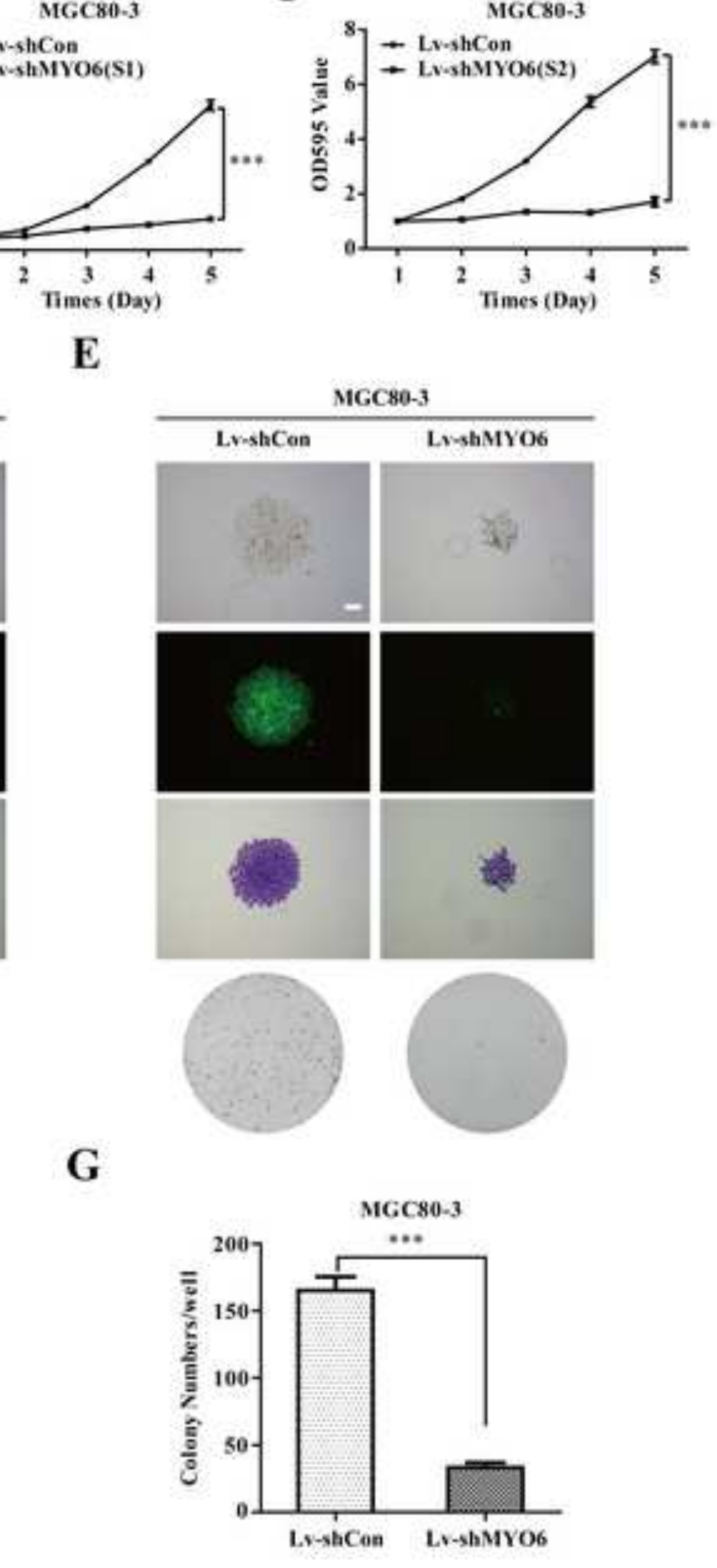

MGC80-3
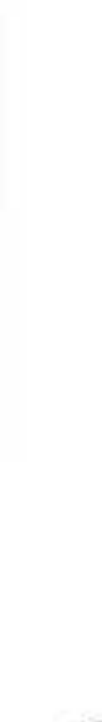
Figure 5

A

AGS

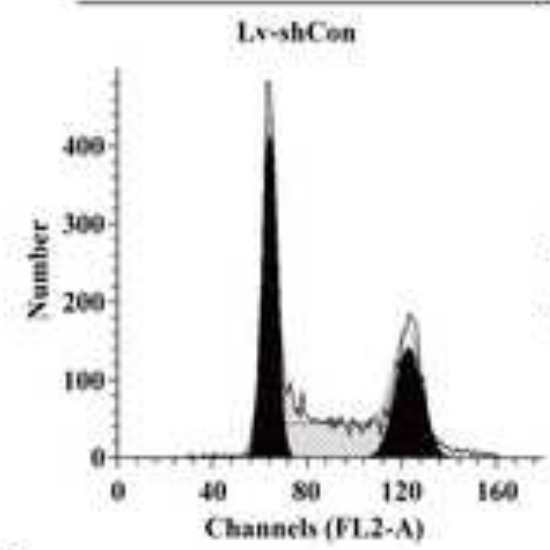

B

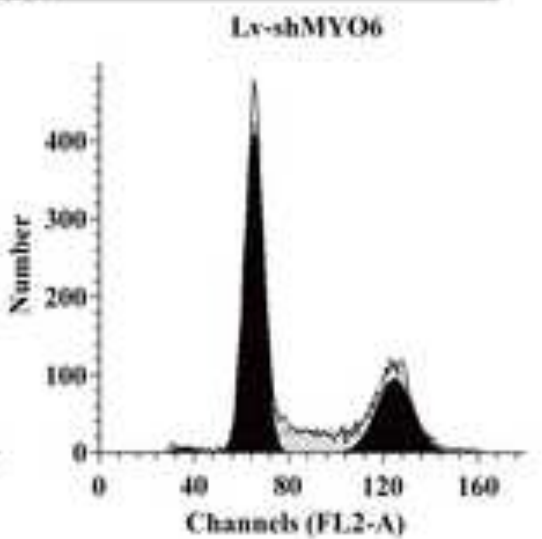

MGCO0-3
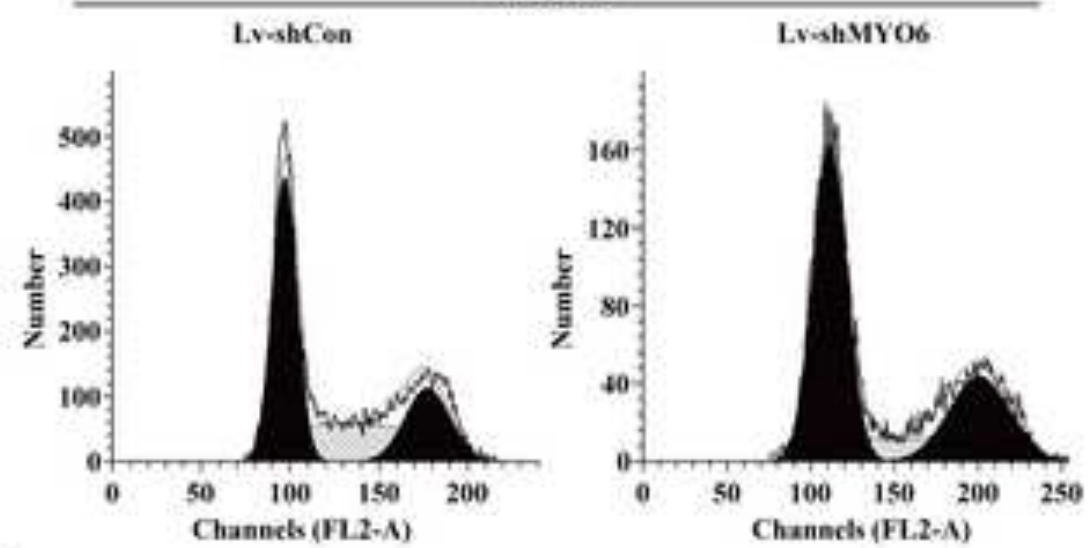

E

$\operatorname{MGCs0-3}$

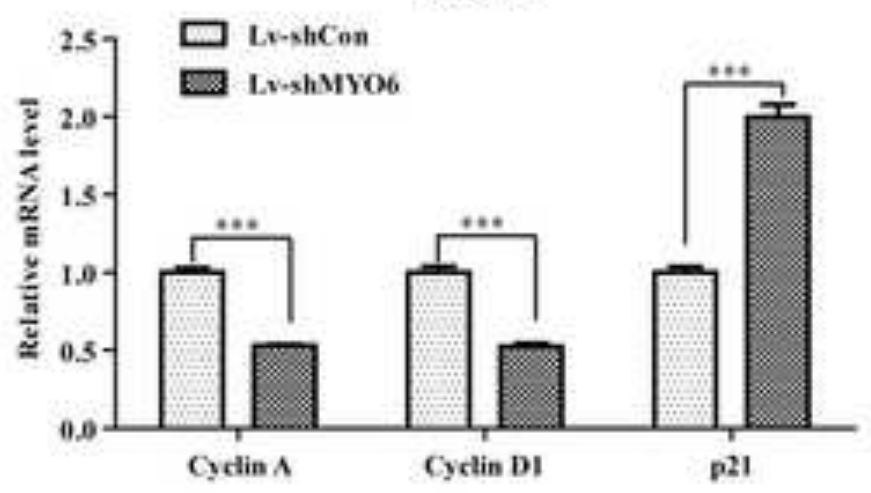

C

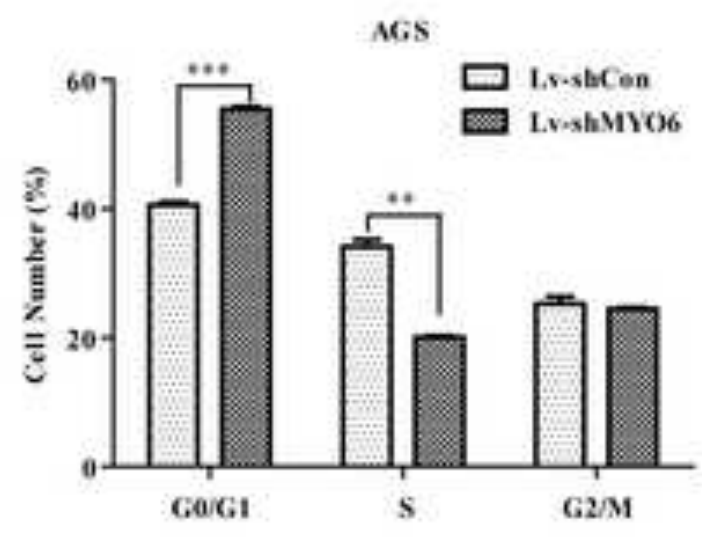

D

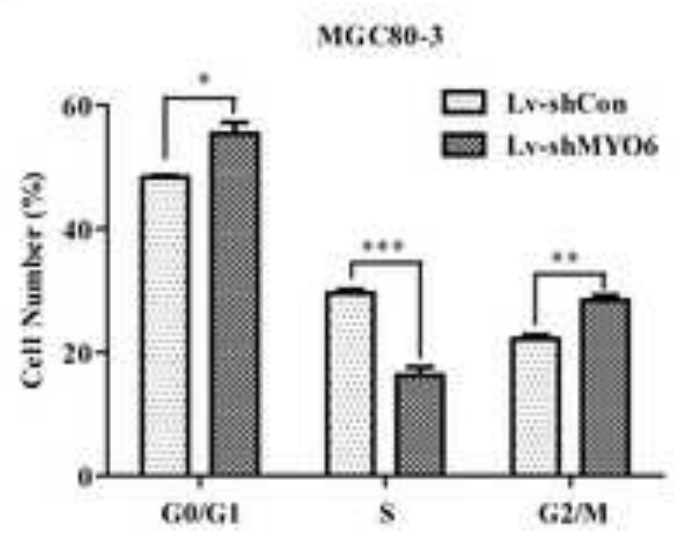

F

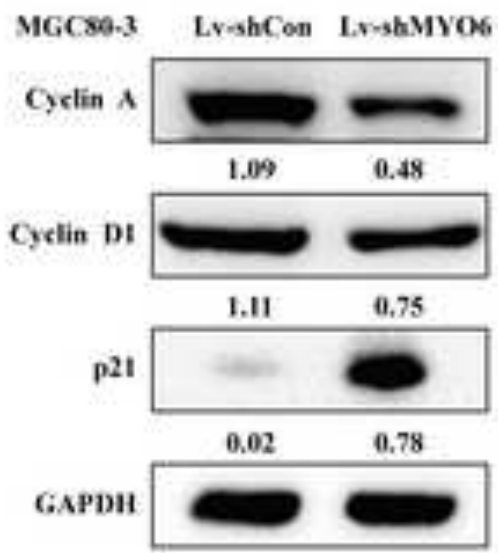


A

AGS

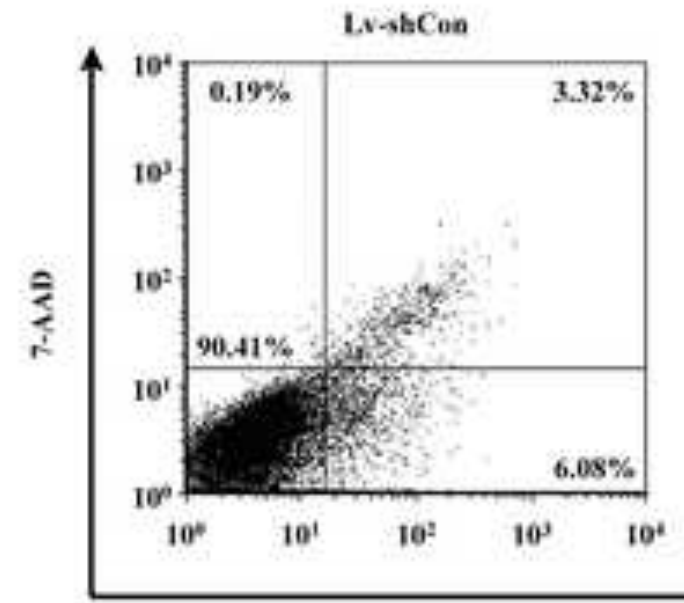

Annexin V-APC

B

AGS

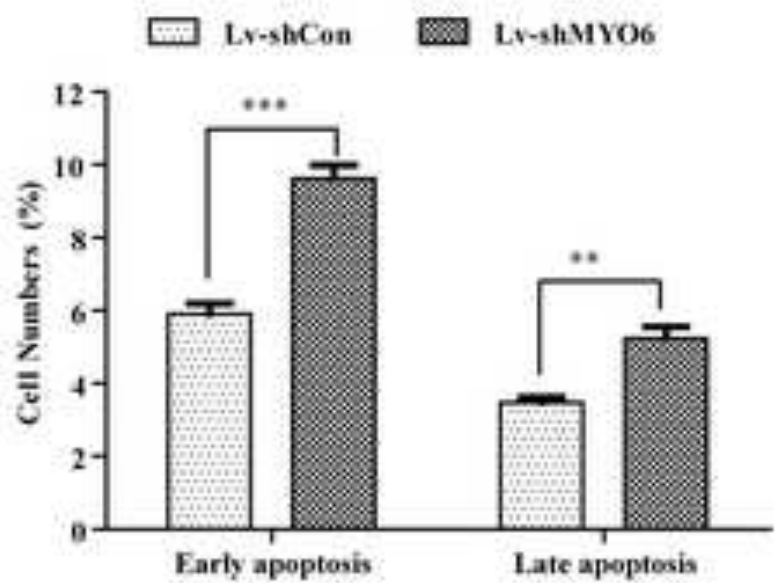

C

1C.C $80-3$

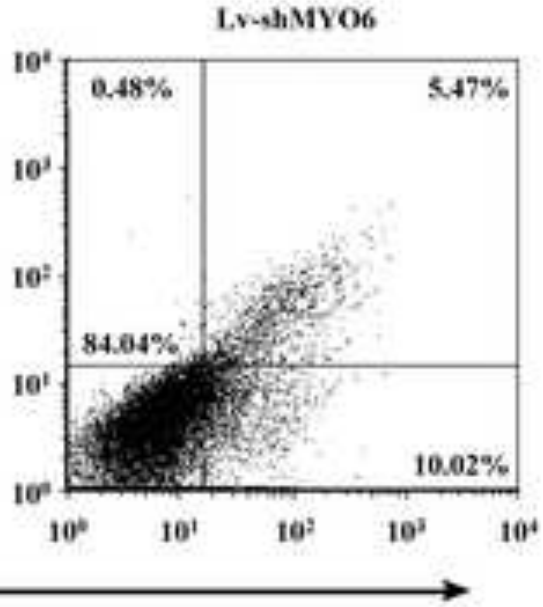

D
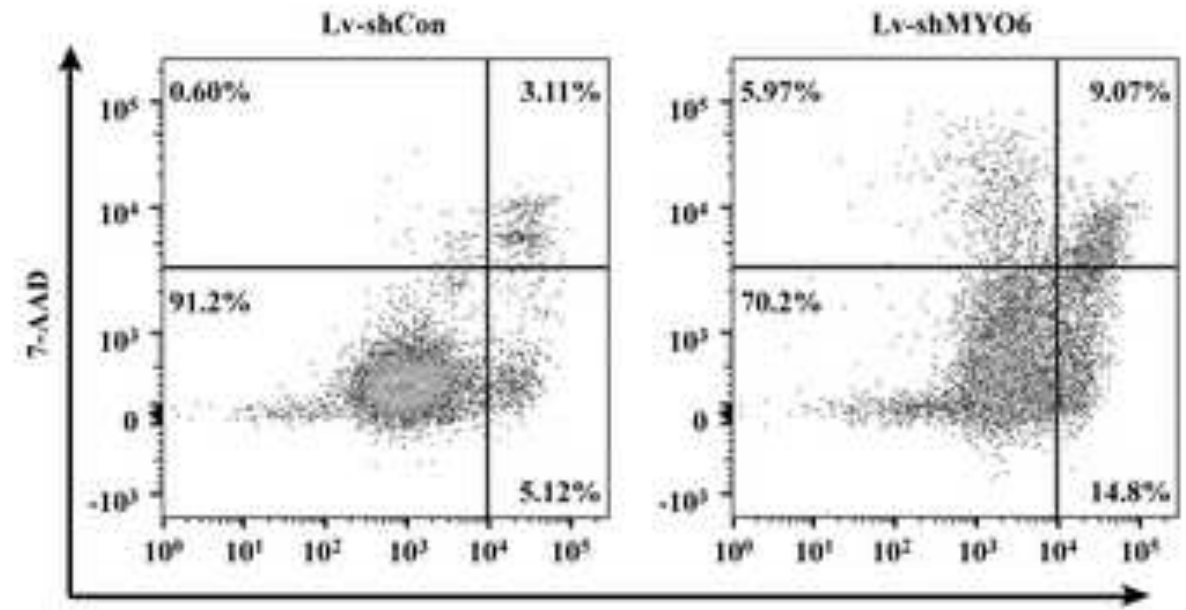

Annexin V-APC

E
MGCso-3

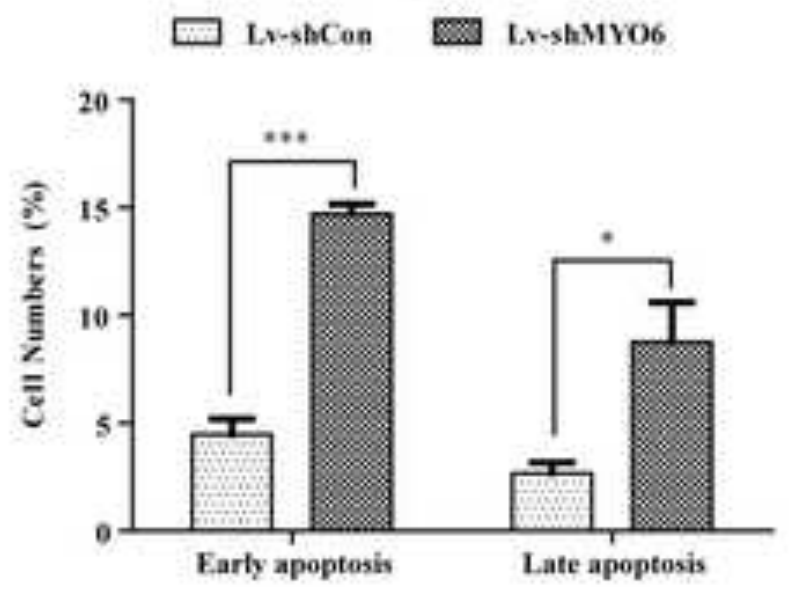

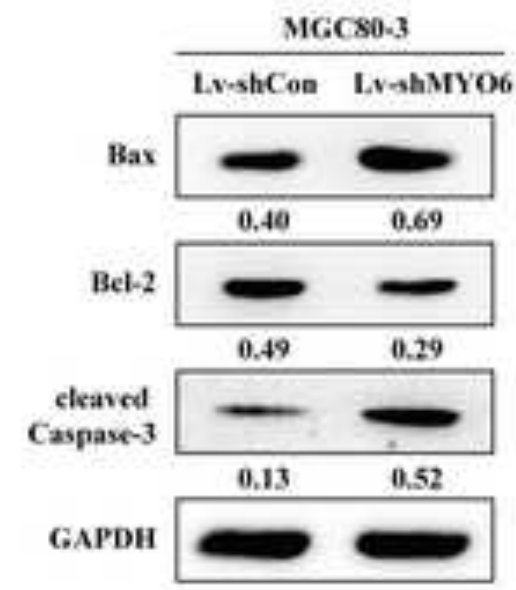


A

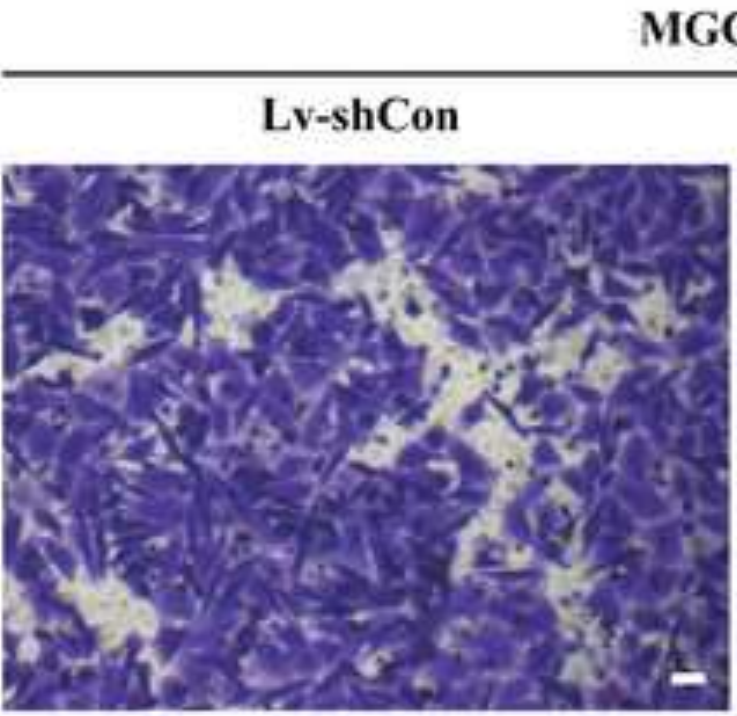

B

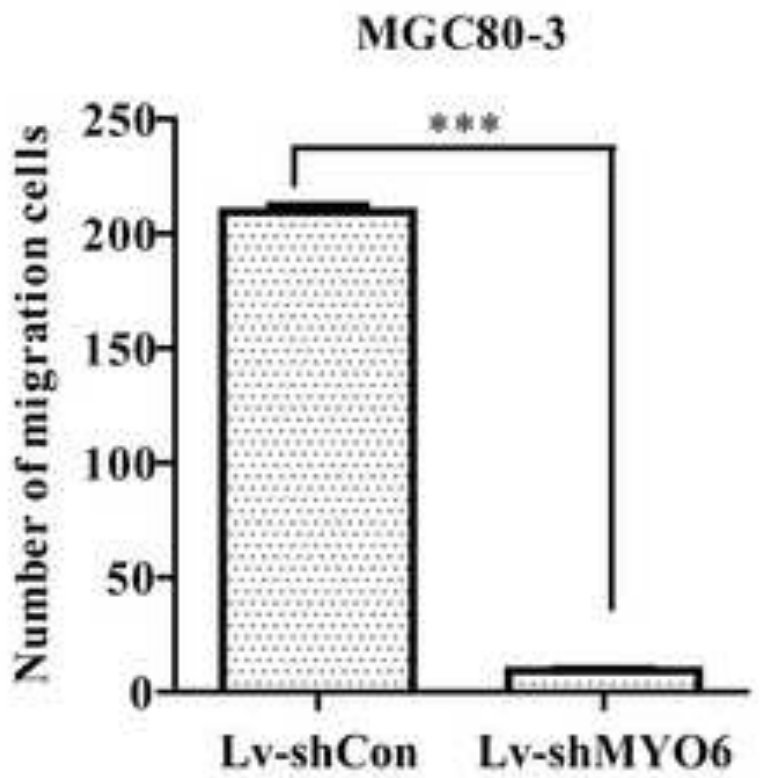

